\title{
Factorial Experiment on Route Structures Using SAAM on a Specified Area in ASEAN
}

\author{
S.M.Phyoe ${ }^{1}$, J.W. Goh ${ }^{2}$, R.Guo ${ }^{3}$, Z.W. Zhong ${ }^{4}$ \\ ${ }^{1,2,3,4}$ Air Traffic Management Research Institute, Nanyang Technological University, Singapore
}

\begin{abstract}
Due to a rapid growth in the air transport industry, ASEAN (Association of Southeast Asia Nations) region has experienced a surge in the air traffic movement in recent years. Several forecasting papers foretell to have the continuous traffic growth for ASEAN region [1,2]. The pilot study of ATS routes forecasts also conclude that there is a need to relook at the current route structures in selected areas in ASEAN [3]. The interaction between the structure of ATS routes and the number of intersections has been studied by using the number of conflicts [4]. This paper will serve as a continuation of the previous study, and the objective is to determine whether there is any consistency in the conclusion when the selected area is changed, using the methodology proposed.
\end{abstract}

\section{Introduction}

The exponential growth in the air traffic movement has left many researchers scrambling for solutions and improvements in the past decades. There are many proposed measures to counterbalance the acceleration, from straightforward solutions like expanding ground infrastructures to sophisticated concepts like the ground delay program [5-7]. The demand for each ATS route also increases. Optimization of en route flow was studied by $\mathrm{Li}$ et al [8]. Integer programming was employed for better traffic flow and follow the suitable ATS routes [8].

Our previous study has investigated the potential factors which might lead to an improvement in the current structure of some ATS routes by using the number of conflicts [4]. A conflict is when two or more aircraft experience a loss of minimum separation [9]. There are several studies on conflict resolution since this is directly related to aircraft safety.

Tomlin et al. created a methodology for two aircraft conflict resolution maneuvers based on reachable sets for hybrid systems with nonlinear dynamic within each discrete state [10]. Wang et al. established a mathematical model for resolving air traffic conflicts [11]. Ma et al. resolved the 3 flights conflict through combining the ADS-B reports with the track optimization and Genetic Algorithm [12]. According to Hong et al, the particle swarm optimization (PSO) was used to solve the nonlinear optimal conflict resolution and flow management problem in their study [13]. Han et al. proposed the potential field differential evolution (PFDE). The differential evolution algorithm was used to optimize the potential field (PF) and proved that PFDE performed better than PF [14]. Matsuno et al. proposed the nearoptimal control method to resolve the aircraft conflict based on the polynomial chaos expansion and pseudospectral methods [15]. Due to the nature of conflicts reduction from our ATS routes improvement, it can also be one of the solutions for conflict resolutions.

However, the results and conclusions are restricted to that particular airspace and are not a representation of the general airspace. In this paper, using the concept of factorial experiment, we will investigate another area in ASEAN and determine if the same conclusion can be obtained.

\section{Factorial experiment - factors and levels}

There are a total of two factors and three levels for the setup of this factorial experiment. They are defined as follows:

1. Structure of the ATS routes ( Figure 1)

1.1. Bidirectional (Bi)

1.2. Unidirectional clockwise (Uc)

1.3. Unidirectional counterclockwise (Ucc)

2. Number of Intersections in the Region (Figure 2)

2.1. Low (L): Fewer than 10 intersections in the area

2.2. Medium (M): Between 10-20 intersections in the area

2.3. High $(\mathrm{H})$ : More than 20 intersections in the area

\section{Methodology}

The methodology follows closely to that done in the previous study [4].

Firstly, an area with high traffic density was selected. This study chose the area of the experiment based on the ASEAN's traffic density which was calculated by using 
SAAM (System of Traffic Assignment and Analysis at a Macroscopic Level) and 2016 June second day traffic data from INNOVATA.

As shown in Figure 3, the airspace was divided into small density cells and the color of the cells indicates the density of the traffic for that area. Since red color indicates the highest density, the particular area covered by red cells was chosen for this study.

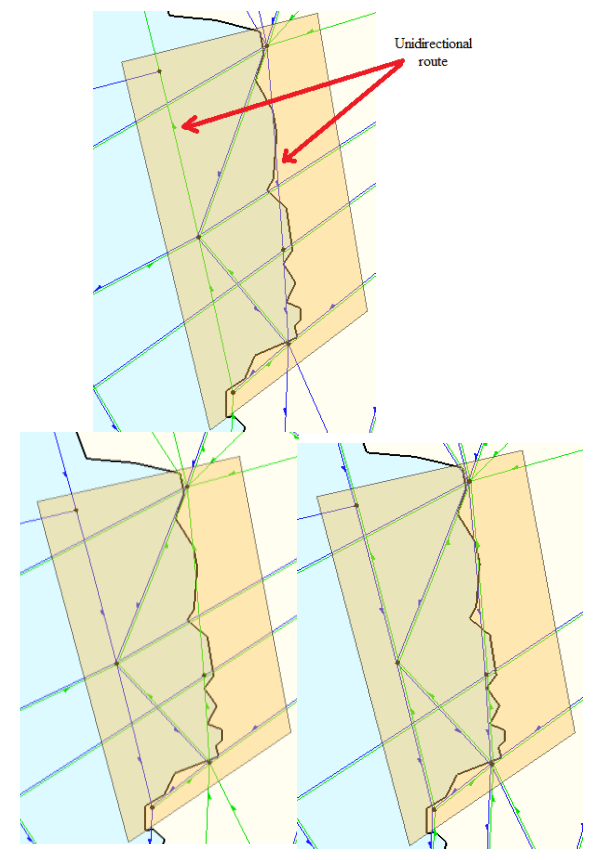

Figure 1. (From top, moving clockwise): Unidirectional clockwise route structure, bidirectional route structure and unidirectional counterclockwise route structure for the chosen ATS routes. The area of concern is shaded in orange.

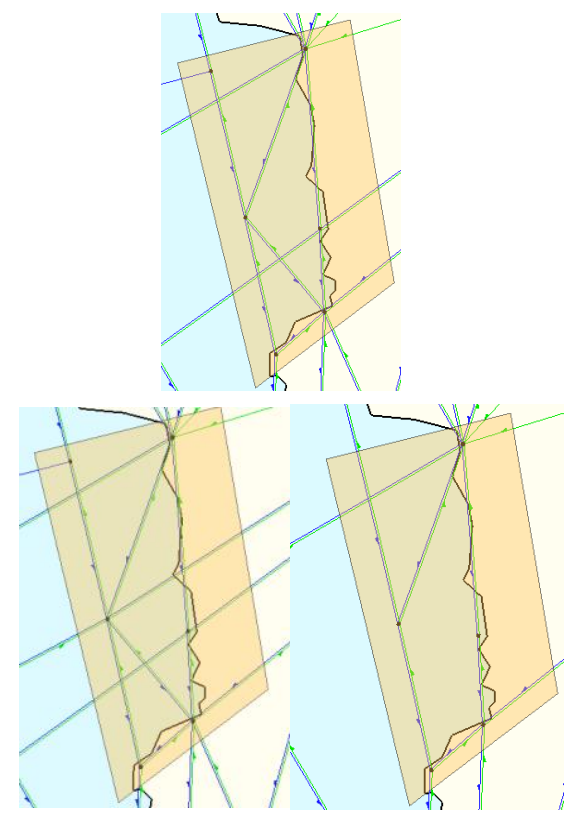

Figure 2. (From top, moving clockwise): Medium number of intersections ( 10 intersections), low number of intersections ( 5 intersections) and high number of intersections (16 intersections). The area of concern is shaded in orange.

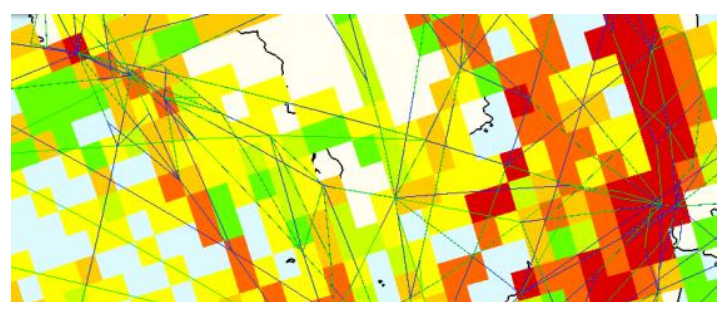

Figure 3. Example 3D Density based on 2016 June second day Traffic (INNOVATA)

Subsequently, two ATS routes were focused based on the extracted whole day information within the area. Following that, the structures of the ATS routes were modified as shown in Figure 1.

The introduction and removal of segments for the intersection modifications were done in a manner not to make the aircraft experience any major rerouting. Figure 2 demonstrates how certain segments of the ATS routes were added or removed.

Once the setup was completed, each scenario was ran thrice using SAAM. Then the number of conflicts was recorded. Finally, the interactions between the factors were studied, and the factorial ANOVA test was performed to determine if there was any difference in conflict rate due to the changes in route structures, the number of intersections and the interaction between route structures and intersections. The three different tests are shown below with their respective decision rules, tested at $5 \%$ level of significance.

1. $\mathrm{H}_{0}: \mu_{\mathrm{Bi}}=\mu_{\mathrm{Uc}}=\mu_{\mathrm{Ucc}}$

$\mathrm{H}_{1}: \mu_{\mathrm{Bi}} \neq \mu_{\mathrm{Uc}} \neq \mu_{\mathrm{Ucc}}$

Decision rule: $\mathrm{F}_{\alpha}(2,18)=3.555$

2. $\mathrm{H}_{0}: \mu_{\mathrm{H}}=\mu_{\mathrm{M}}=\mu_{\mathrm{L}}$

$\mathrm{H}_{1}: \mu_{\mathrm{H}} \neq \mu_{\mathrm{M}} \neq \mu_{\mathrm{L}}$

Decision rule: $\mathrm{F}_{\alpha}(2,18)=3.555$

3. $\mathrm{H}_{0}$ : interaction is absent

$\mathrm{H}_{1}$ : interaction is present

Decision rule: $\mathrm{F}_{\alpha}(4,18)=2.928$

\section{Results and analysis}

Table 1 records the number of conflicts after running each scenario thrice with SAAM.

Table 2 shows the values of the sum of squares (SS), degree of freedom $(\mathrm{dF})$, mean squared (MS) and the Fvalue $(\mathrm{F})$ based on the results in Table 1.

Table 1: The number of conflicts recorded after running each scenario thrice.

\begin{tabular}{|l|l|l|l|}
\hline & Low & Medium & High \\
\hline \multirow{3}{*}{ Bidirectional } & 10 & 19 & 15 \\
\cline { 2 - 4 } & 9 & 21 & 18 \\
\cline { 2 - 4 } & 17 & 41 & 48 \\
\hline \multirow{2}{*}{$\begin{array}{l}\text { Unidirectional } \\
\text { clockwise }\end{array}$} & 6 & 13 & 11 \\
\cline { 2 - 4 } & 11 & 23 & 11 \\
\cline { 2 - 4 } & 8 & 14 & 10 \\
\hline \multirow{2}{*}{$\begin{array}{l}\text { Unidirectional } \\
\text { counterclockwise }\end{array}$} & 16 & 32 & 34 \\
\cline { 2 - 4 } & 13 & 36 & 43 \\
\cline { 2 - 4 } & 4 & 7 & 28 \\
\hline
\end{tabular}




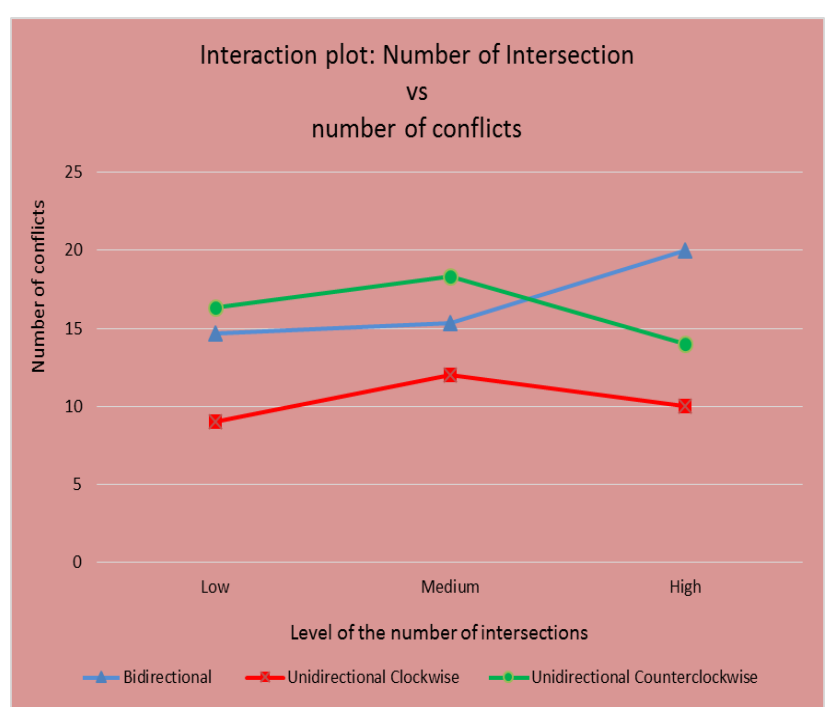

Figure 4. The interaction plot between intersections and conflicts

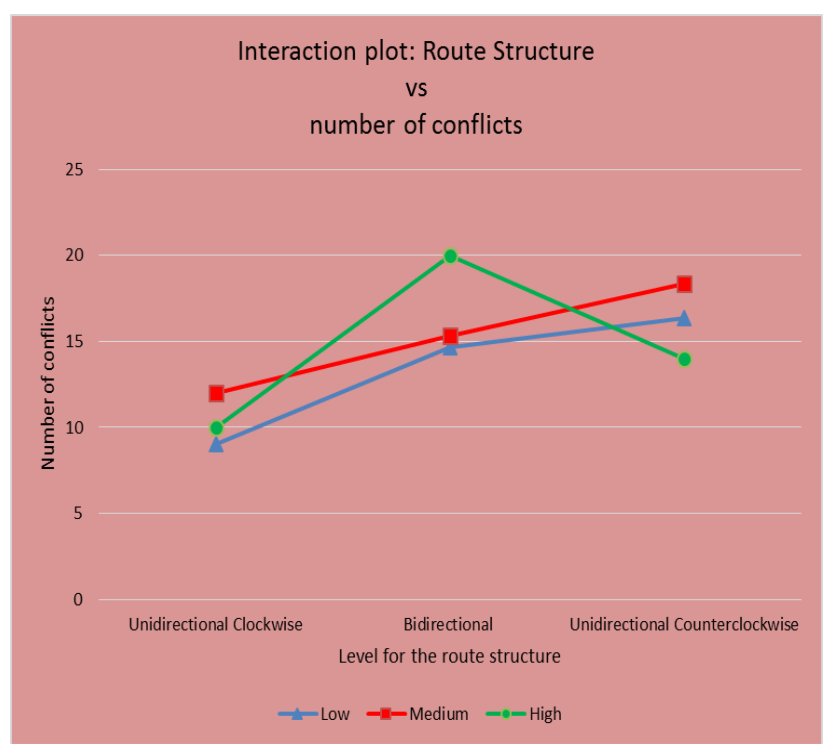

Figure 5. The interaction plot between route structures and the numbers of conflicts.

Figures 4 and 5 interpret that there is some form of interaction between the route structure and the number of intersections considering the plots are nonparallel. As shown in Figure 4, the plot lines for the unidirectional routes (both clockwise and counterclockwise) are more parallel comparing to the plot line for the bi-directional route, revealing that the effect of interaction is more significant for the bidirectional route structures.

As shown in Figure 5, the plot lines for intersections are nonparallel for all the three levels of intersections (low, medium \& high). This observation supports the observation mentioned above for Figure 4, and thus it is deduced that the effect of the interaction is affected by the route.
Table 2. The numerical values for sum of squares, degree of freedom, mean squared and the F-value for each row

\begin{tabular}{|l|l|l|l|l|}
\hline & SS & dF & MS & $\begin{array}{l}\text { F- } \\
\text { value }\end{array}$ \\
\hline $\begin{array}{l}\text { Structure of the ATS } \\
\text { routes (RS) }\end{array}$ & 731.19 & 2 & 365.59 & 3.62 \\
\hline $\begin{array}{l}\text { Number of Intersections } \\
(\mathrm{NOI})\end{array}$ & 49.85 & 2 & 24.93 & 0.25 \\
\hline Interaction $(\mathrm{RS} \times \mathrm{NOI})$ & 1325.70 & 4 & 331.43 & 3.28 \\
\hline Error & 1817.33 & 18 & 100.96 & - \\
\hline Total & 3924.07 & 26 & - & - \\
\hline
\end{tabular}

Numerically, Figure 5 reveals that when the number of interactions is high, employing the unidirectional route structure (both clockwise and counterclockwise) encounters less conflicts than the bidirectional route structure. Whereas, for low number of interactions, there are least conflicts by applying unidirectional clockwise route structure. In conclusion, among the three different structures, the unidirectional clockwise route structure yields the lowest conflicts for all levels. This is the different result since the bidirectional route structure was the lowest in our previous study. However, this information is insufficient to demonstrate that the change in the structure of the selected ATS routes indeed results in a change in a number of conflicts.

According to Table 2, since $\mathrm{F}_{\mathrm{RS}} \geq \mathrm{F}_{\alpha}$, the null hypothesis is rejected and it is concluded that there is a difference in the number of conflicts when there is a change in the structure of the selected ATS routes. On the other hand, since $\mathrm{F}_{\mathrm{NOI}} \leq \mathrm{F}_{\alpha}$, the null hypothesis is not rejected and it is concluded that there is no significant difference in the number of conflicts when there is a change in the number of intersections in a region. Likewise, the null hypothesis is rejected since $\mathrm{F}(\mathrm{RS} \times$ NOI) $\geq F_{\alpha}$, and it is concluded that the number of conflicts can be reduced with a modification of both the selected ATS routes structure and the number of intersections between routes in the targeted region.

The results of the factorial ANOVA test suggest that the number of intersections has no much influence on the number of conflicts. However the structure of the selected ATS routes, particularly their cruising directions, can be one of the primary factors that can improve the airspace or the route capacity of this selected area. In addition, the interaction between route structures and intersections can be another element to reduce conflicts and improve the airspace. It is critical to note that the above results are restricted to this particular area and may not apply to every case since the arrangement of the ATS routes differs in every country.

\section{5 conclusion}

From the results, the main conclusion is that the number of conflicts could be significantly affected by the 
direction of routes for this selected area and the interaction of the routes' direction and intersection. It can also conclude statistically that for this selected area, there is no significant difference in the number of conflicts if we change the number of intersections itself. In comparison with the previous result, the impact of the routes' direction remains the same, but the interaction of the routes' direction and the intersection is found to be the new influence factor. Therefore, we can assume that the effect on the number of conflicts also depend on the regions. By applying the methodology, the service providers can design the optimal route structures for their targeted airspace to achieve less number of conflicts. In future, more advanced and detailed study for the factorial ANOVA test will be conducted and the reason of different performance for different regions will be discussed.

\section{Acknowledgment}

This research was sponsored by the ATMRI of NTU and CAAS via ATMRI Project No. 2014-D2-ZHONG for Regional Airspace Capacity Enhancement - ASEAN Pilot.

\section{References}

1. Airbus S.A.S (2015), Airbus Global Market Forecast 2015-2034. [Online]. Available: http://www.airbus.com/company/market/forecast/

2. S.M. Phyoe, Y.X. Lee, Z.W. Zhong, Determining the Future Demand: Studies for Air Traffic Forecasting, International Journal of Technology and Engineering Studies, Vol 2, No.3, pp. 83-86, (2016)

3. D. Raheja, Y.X. Lee, Z.W. Zhong, Utilizing Regression Algorithm for ATS Routes Forecasts, Singapore 34th International Conference on Engineering \& Technology, Computer, Basic and Applied Sciences (ECBA-2017), Singapore, Jul (2017)

4. J.W. Goh, Y.X. Lee, S. Aneeka, S.M. Phyoe, Z.W. Zhong, Factorial Experiment on Route Structures leveraging on Current Air Traffic Management Technology on Selected Area in ASEAN, Singapore 28th International Conference on Engineering \& Technology, Computer, Basic and Applied Sciences (ECBA-2017), Singapore, Feb (2017)
5. E. P. Gilbo, Optimizing airport capacity utilization in air traffic flow management subject to constraints at arrival and departure fixes, IEEE Transactions on Control Systems Technology, Vol 5, pp. 490, (1997)

6. R.L. Hoffman, M.O. Ball, Measuring Ground Delay Program Effectiveness Using the Rate Control Index, Journal of Air Traffic Control, Vol 42, No.2, pp. 1923, (2000)

7. M. Ball, G. Lulli, Ground Delay Programs: Optimizing over the Included Flight Set Based on Distance, Air Traffic Control Quarterly, Vol 12, No.1, pp. 1-25, (2004)

8. Q. Li, Y. Zhang and R. Su, "A Flow-based Flight Scheduler for En-route Air Traffic Management", IFAC-PapersOnLine, vol. 49, no. 3, pp. 353-358, 2016.

9. J. Kuchar and L. Yang, "A review of conflict detection and resolution modeling methods", IEEE Transactions on Intelligent Transportation Systems, vol. 1, no. 4, pp. 179-189, 2000.

10. C. Tomlin, G. Pappas and S. Sastry, "Conflict resolution for air traffic management: a study in multiagent hybrid systems", IEEE Transactions on Automatic Control, vol. 43, no. 4, pp. 509-521, 1998.

11. K. Wang and R. Nie, "Research on Detection and Resolution Model of Air Traffic Conflict", in EProduct E-Service and E-Entertainment (ICEEE), Henan, China, 2010.

12. Y. Ma, Y. Ni and P. Liu, "Aircrafts Conflict Resolution Method Based on ADS-B and Genetic Algorithm," 2013 Sixth International Symposium on Computational Intelligence and Design, Hangzhou, 2013, pp. 121-124.

13. Y. Hong, B. Choi, G. Oh, K. Lee and Y. Kim, "Nonlinear Conflict Resolution and Flow Management Using Particle Swarm Optimization", IEEE Transactions on Intelligent Transportation Systems, pp. 1-10, 2017.

14. D. Han, X. Zhang and X. Li, "Aircrafts Conflict Resolution Using Differential Evolution", in 12th World Congress on Intelligent Control and Automation (WCICA), Guilin, China, 2016.

15. Y. Matsuno, T. Tsuchiya and N. Matayoshi, "NearOptimal Control for Aircraft Conflict Resolution in the Presence of Uncertainty", Journal of Guidance, Control, and Dynamics, vol. 39, no. 2, pp. 326-338, 2016. 